\title{
Effect of Energetic Electron Irradiation on Graphene
}

\author{
Isaac Childres ${ }^{1,2}$, Romaneh Jalilian ${ }^{1,2}$, Michael Foxe ${ }^{4}$, Alex Chernyshov ${ }^{1,2}$, \\ Leonid Rohkinson $^{1,2}$, Igor Jovanovic ${ }^{4}$, Yong P. Chen ${ }^{1,2,3}$ \\ ${ }^{1}$ Department of Physics, Purdue University, West Lafayette, IN, 47907 \\ ${ }^{2}$ Birck Nanotechnology Center, Purdue University, West Lafayette, IN, 47907 \\ ${ }^{3}$ School of Electrical and Computer Engineering, Purdue University, West Lafayette, IN, 47907 \\ ${ }^{4}$ School of Nuclear Engineering, Purdue University, West Lafayette, IN, 47907
}

\begin{abstract}
Electron beam exposure is a commonly used tool for fabrication and imaging of graphene-based devices. Using Raman spectroscopy and electronic transport measurements, we have studied the effect of prolonged exposure of electron beams on exfoliated graphene on $\mathrm{SiO}_{2} / \mathrm{Si}$ substrates and the performance of electronic devices based on exfoliated graphene. Raman spectra indicate emergence of characteristic defects. Electronic transport measurements show an overall decrease in graphene's conductivity and a shift of the Dirac point. Our results are valuable for understanding the possible defects generated in graphene by electron beam exposure and in high-radiation environment in general.
\end{abstract}

Keywords: Graphene, defects, electron beam, Raman spectroscopy, electron transport PACS: $61.80 . \mathrm{Fe}$, 61.82.Rx, 78.30.Na, 78.70.-g, 81.40.Rs

\section{INTRODUCTION}

Graphene has been the focus of much research in material science and nanotechnology due to its many unique properties and potential in device applications. Many studies report on graphene's very high electrical conductivity $[1,2]$ at room temperature, and it is discussed as having potential for use in next-generation transistors [3] and novel nano-sensors [4].

Here we present a study of extended exposure of energetic electrons on graphene and the effect of this exposure on the conductivity and crystal structure of graphene. Our interest in the change in graphene, especially its degree of degradation, caused by electron bombardment is threefold: 1) electron beams (e-beams) are a commonly used tool in both imaging of graphene, e.g. scanning electron microscopy (SEM) and transmission electron microscopy (TEM), and fabrication of graphene devices using ebeam lithography; 2) such studies are important to develop graphene-based radiationhard electronics which can stand up to extreme conditions such as charged particle irradiation in space; and 3) our group is currently developing an ionizing radiation 


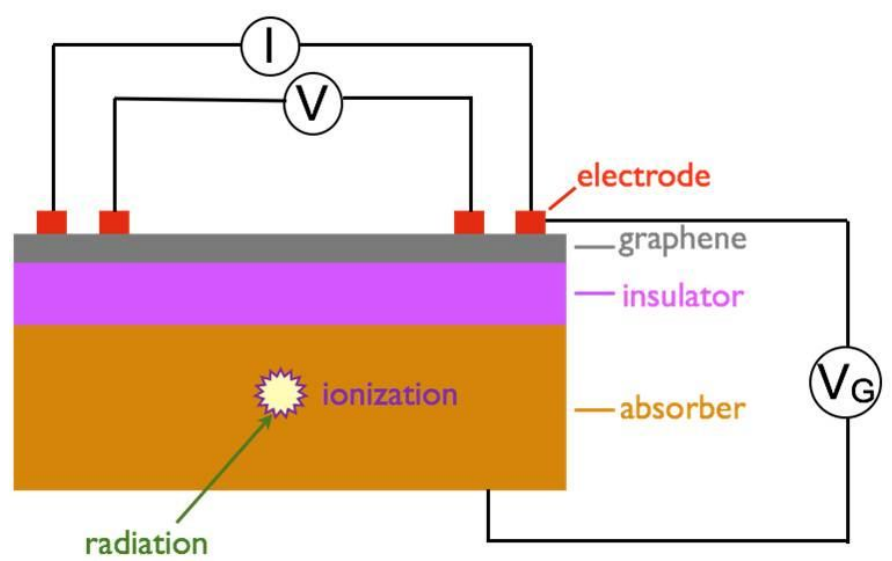

FIGURE 1. Schematic of a graphene field effect transistor used as a radiation detector. Incident radiation (such as gamma-rays) ionize a part of the undoped, electrically biased semiconductor substrate, creating charge carriers that modify the E-field applied on the graphene. A graphene FET could detect this change in the E-field as a change in its resistance.

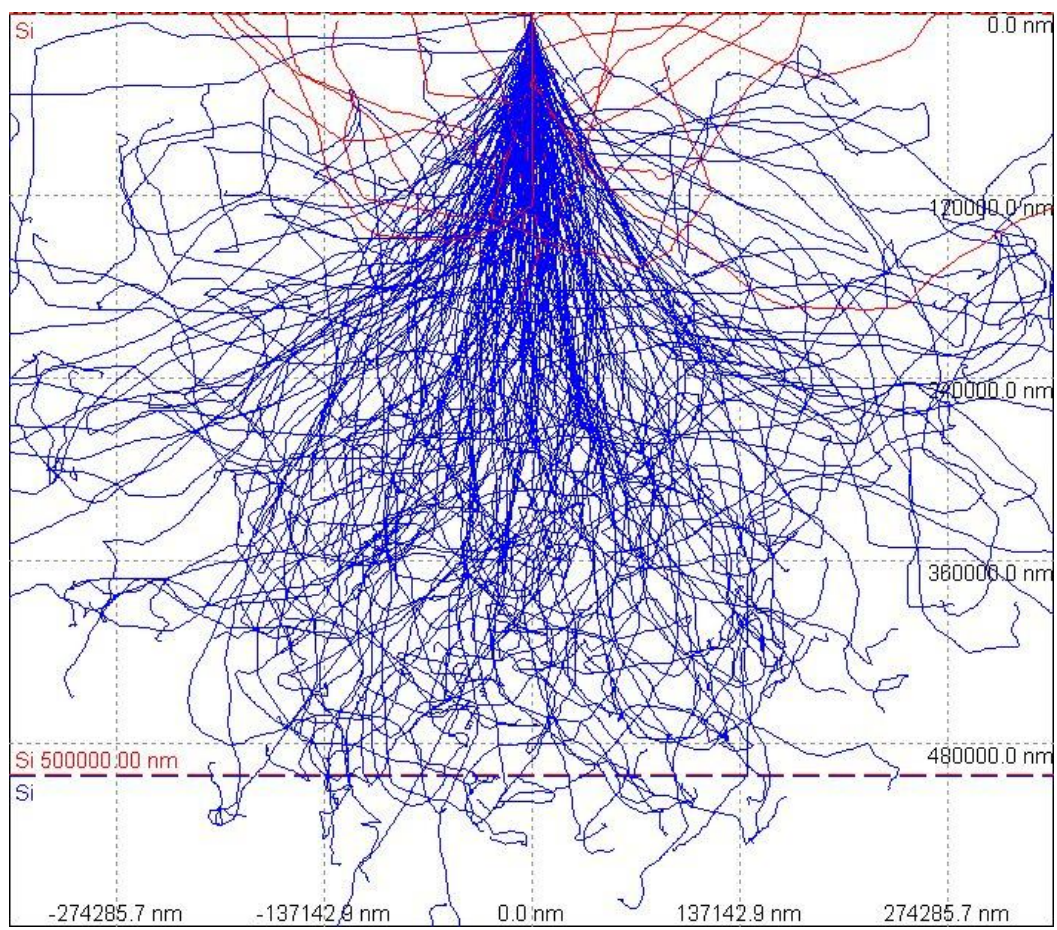

FIGURE 2. Monte Carlo (using CASINO) simulation of possible paths of secondary electrons ionized by a $400 \mathrm{keV}$ Compton electron (e.g. generated by a gamma-ray photon) through a $0.5 \mathrm{~mm}$-thick silicon substrate. When using graphene FETs as radiation detectors, energetic electrons produced by gamma or other ionizing radiation could pass through the substrate and interact with the graphene. 
detector which uses the field effect in graphene, as in Figure 1. Radiation produces primary Compton electrons in the semiconductor substrate supporting graphene. The electrons travel through the substrate, as modeled by Fig. 2, and can reach the surface containing graphene. Therefore, understanding how energetic electrons interact with the graphene is important for the reliability and long-term robustness of such sensors.

\section{METHODS}

We have used micro Raman spectroscopy and electrical characterization to study in detail the effects of e-beam radiation on pristine single-layer exfoliated graphene to understand how disorder develops in the material through bombardment of energetic electrons.

In our experiments, the graphene was exposed to an e-beam in an EVO 40 SEM system with a $0.15 \mathrm{nA}$ beam current at a $30 \mathrm{keV}$ acceleration voltage over a $50 \mu \mathrm{m}$-by$50 \mu \mathrm{m}$ area for 5 minutes. This gives a dosage of $112.5 \mathrm{e}^{-} / \mathrm{nm}^{2}$. The graphene flakes irradiated were exfoliated from natural graphite using Scotch tape onto a highly doped silicon substrate with a $300 \mathrm{~nm} \mathrm{SiO}{ }_{2}$ over-layer [1]. The flakes were single-layer and typically $20-100 \mu \mathrm{m}^{2}$ in size.

\section{Raman Spectroscopy}

A single-layer graphene sample was measured by a confocal DXR Raman system using a $514 \mathrm{~nm}$ laser with $2 \mathrm{~mW}$ power. Before exposure, the Raman spectrum showed the signature for pristine single-layer graphene, with a $\mathrm{G}$ peak at $1584 \mathrm{~cm}^{-1}$ and a $2 \mathrm{D}$ peak at $2674 \mathrm{~cm}^{-1}$ [5], with a ratio of the intensities of the $2 \mathrm{D}$ and $\mathrm{G}$ peaks, $\mathrm{I}(2 \mathrm{D}) / \mathrm{I}(\mathrm{G})$, of 2.62. Prior to e-beam exposure we observed no sign of D or D' peaks, commonly associated with disorder in graphene (Fig. 3).

After e-beam exposure, the Raman spectrum showed an emergence of a D peak at $1341 \mathrm{~cm}^{-1}$ and a small $\mathrm{D}^{\prime}$ peak appeared at $1624 \mathrm{~cm}^{-1}[5]$. The peak ratio $\mathrm{I}(2 \mathrm{D}) / \mathrm{I}(\mathrm{G})$ shrunk to 2.32 , while $\mathrm{I}(\mathrm{D}) / \mathrm{I}(\mathrm{G})$ changed to 0.565 and $\mathrm{I}(\mathrm{D}) / \mathrm{I}(2 \mathrm{D})$ changed to 0.244 .

The increase of $\mathrm{I}(\mathrm{D}) / \mathrm{I}(\mathrm{G})$ is attributed to a gradual evolution from the $\mathrm{sp}^{2}$-bonded carbon found in graphene into amorphous carbon with substantial $\mathrm{sp}^{3}$ bonding [5]. Our results are also consistent with similar Raman work done by Teweldebrhan and Baladin [6]. 


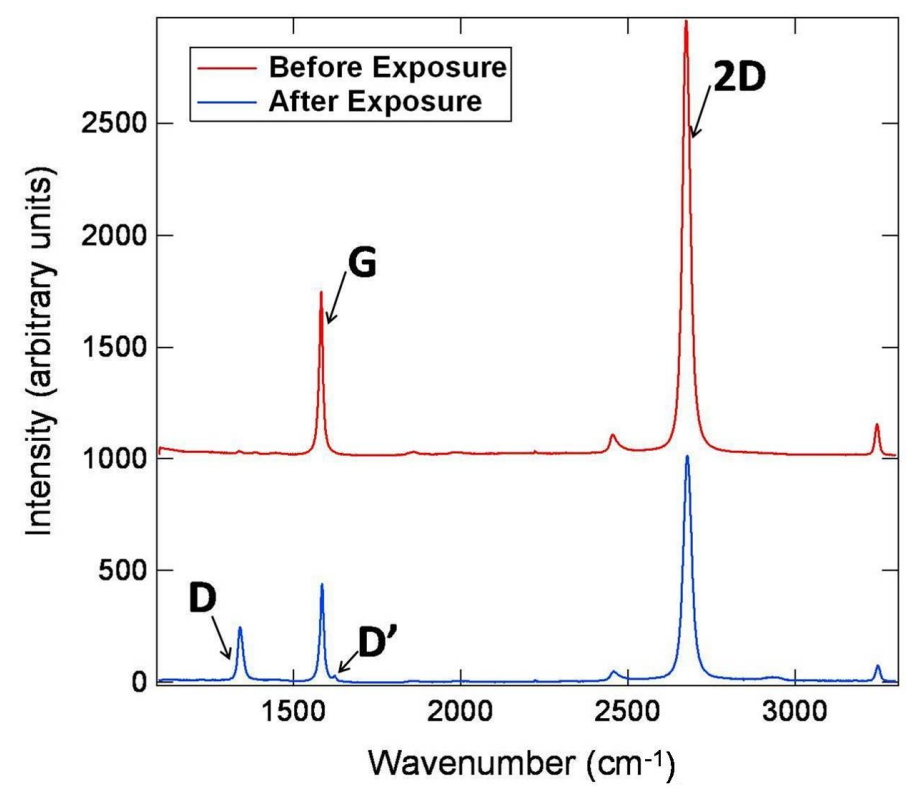

FIGURE 3. Raman spectrum measured by a $514 \mathrm{~nm}$ laser operating at $2 \mathrm{~mW}$ on a single-layer graphene sample before and after exposure to e-beam radiation. Exposure causes a reduction of both the $\mathrm{G}(1584$ $\left.\mathrm{cm}^{-1}\right)$ and $2 \mathrm{D}\left(2674 \mathrm{~cm}^{-1}\right)$ peaks while causing an emergence of $\mathrm{D}\left(1341 \mathrm{~cm}^{-1}\right)$ and $\mathrm{D}^{\prime}\left(1624 \mathrm{~cm}^{-1}\right)$ peaks.

The $\mathrm{D}$ and $\mathrm{D}^{\prime}$ peaks are clear indications of disorder in the graphene.

\section{Electrical Measurements}

The electrical characterization, performed on three single-layer samples, was a 2terminal conductance measurement under a varying back gate voltage across the $\mathrm{Si}$ substrate. A typical graphene device used in these studies is shown in Fig. 4. Graphene is a zero-gap semiconductor/semimetal: as the back gate voltage is varied, there is a voltage value where the majority charge carrier density shifts from p-type to n-type. This is the point of lowest conductivity, because the average charge density is zero. In addition to measuring an overall change in conductance, we also found a shift of this "charge-neutral point" (CNP) after the e-beam exposure.

Before e-beam irradiation, we measured CNPs of $24 \mathrm{~V}, 25 \mathrm{~V}$ and $26.5 \mathrm{~V}$ for three single-layer samples. These samples typically have a positive CNP because of $\mathrm{p}$ doping caused by environmental impurities such as water. After exposure, the CNP of all samples shifted toward the negative $(14 \mathrm{~V}, 4 \mathrm{~V}$, and $5 \mathrm{~V}$ respectively). In each case, there is also an overall decrease of conductivity, especially at the CNP, which can be seen in Fig. 5.

We interpret these results as due to a build-up of trapped holes at the $\mathrm{Si} / \mathrm{SiO}_{2}$ interface, following the generation of electron-hole pairs (ionized by the energetic ebeam irradiation) in the substrate: the electrons created are more mobile and can leave 
the sample quickly (eg. through the gate or contacts) while the less mobile, trapped holes will induce n-type carriers (electrons) in the graphene, thus lowering the (positive) CNP (voltage required to turn the graphene from p-type to n-type). This is similar to the mechanisms for the well-known reduction of threshold voltages in irradiated MOSFET [7]. Repeated measurements after the irradiation reveals a slow (in the course of days) positive shift of the (negatively-shifted) CNP back toward its original pre-radiation value, possibly as a result of the trapped holes slowly leaking from sample. We are currently investigating the phenomenon in more detail.

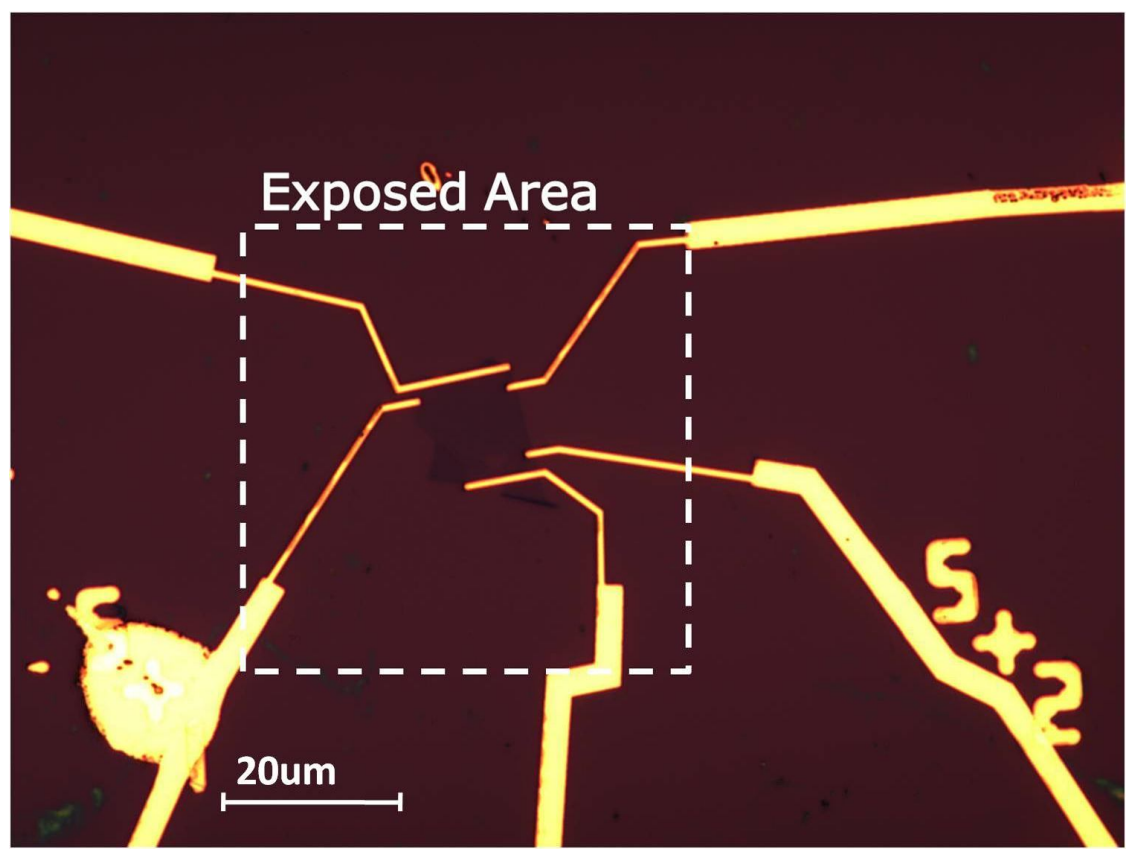

FIGURE 4. Graphene FET device showing the area of the surface exposed to $112.5 \mathrm{e}^{-} / \mathrm{nm}^{2}$ of $30 \mathrm{keV}$ electron radiation.

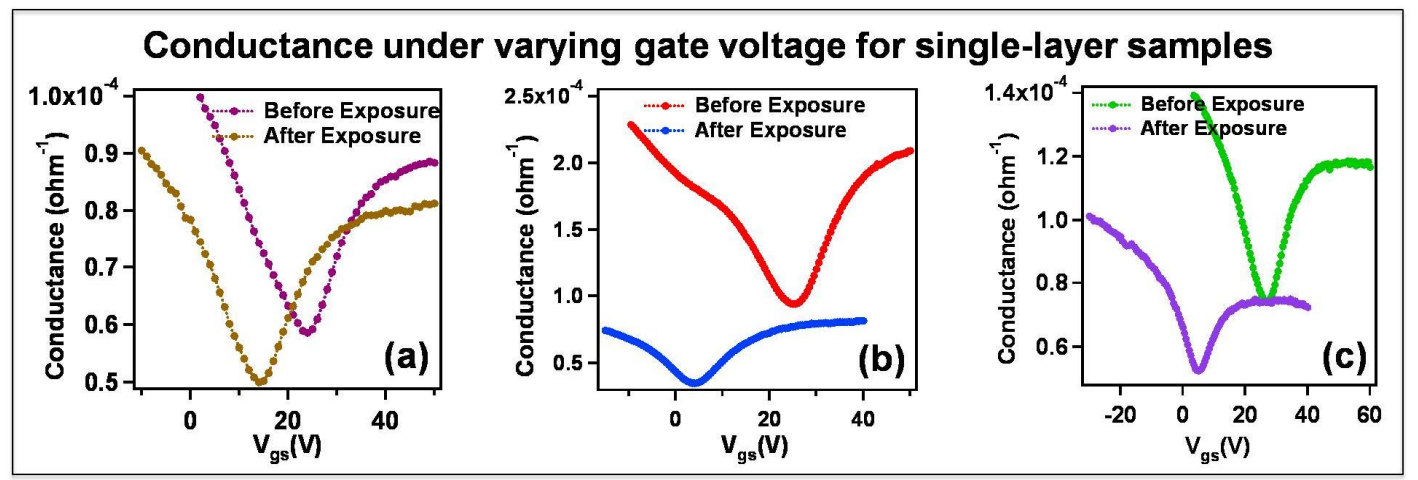

FIGURE 5. Gate voltage $\left(\mathrm{V}_{\mathrm{gs}}\right)$-dependent conductance measurements made on 3 single-layer graphene samples with a $10 \mathrm{mV}$ drain voltage $\left(\mathrm{V}_{\mathrm{ds}}\right)$ for the first sample (a) and $5 \mathrm{mV}$ drain for the others $(\mathrm{b}, \mathrm{c})$ both before and after e-beam exposure. Conductance was obtained from the measured 2-terminal current 
$\left(\mathrm{I}_{\mathrm{ds}}\right.$ ) divided by $\mathrm{V}_{\mathrm{ds}}$. The conductance at the charge-neutral point decreased from: (a) $5.86 * 10^{-5} \Omega^{-1}$ before exposure to $5.00 * 10^{-5} \Omega^{-1}$ after exposure; (b) $9.41 * 10^{-5} \Omega^{-1}$ to $3.50 * 10^{-5} \Omega^{-1}$; (c) $7.40^{*} 10^{-5} \Omega^{-1}$ to $5.25 * 10^{-5} \Omega^{-1}$.

The decrease of conductivity is consistent with the development of disorder peaks in the Raman spectrum, as defects in the carbon lattice can also cause more carrier scattering.

\section{CONCLUSION}

In this study, we have shown evidence of disorder and conductivity reduction caused by energetic electron irradiation of graphene. Disorder and a development toward

amorphous carbon can be seen in the Raman spectroscopy data taken after the exposure, and the conductivity reduction is evident from the electrical characterization. We conclude that care should be taken when using e-beam-based lithography or imaging on graphene, as prolonged exposure could cause a degradation of graphene properties. The study is also relevant for the development of radiation-hard electrons and radiation sensors based on graphene.

\section{REFERENCES}

1. A.K. Geim and K.S. Novoselov, Nature Materials 6, 183-191 (2007)

2. C. Berger, et. al., Science 312, 1191-1196 (2006)

3. M.C. Lemme, et. al., IEEE Electron Device Letters 28, 282-284 (2007)

4. F. Schedin, et. al., Nature Materials 6, 652-655 (2007)

5. A.C. Ferrari, et. al., Physical Review Letters 97, 187401 (2006)

6. D. Teweldebrhan and A.A. Baladin, Applied Physics Letters 94, 013101 (2009)

7. T. P. Ma and Paul V. Dressendorfer, Ionizing radiation effects in MOS devices and circuits, Wiley (1989) 